\title{
Spatial Wave Function Diminishing Due to Friction Force
}

\author{
Arafa Ahmed Mohamed Yagob ${ }^{1,2}$, M. Dirar Abd Allah ${ }^{3}$, Kh.M. Haroun ${ }^{4}$ \\ ${ }^{1}$ Department of Medical Physics, College of Medicine, Jazan University, Saudi Arabia \\ ${ }^{2}$ Department of Physics and Mathematics, College of Education, Kassala University, Sudan \\ ${ }^{3}$ Department of Physics, College of Science, Sudan University of Science and Technology, Sudan \\ ${ }^{4}$ Department of Physics, College of Education, Elzeiim Elazhari University, Sudan
}

\begin{abstract}
Schrodinger equation for resistive medium is obtained through momentum operator. This new equation reduces to ordinary Schrodinger equation in the absence of friction. This equation describes spatially decaying wave function when particles are treated as strings. This solution can describe the inelastic scattering process.
\end{abstract}

Keywords: Resistive medium, friction, momentum, string theory, inelastic scattering

\section{Introduction}

Quantum mechanics is the body of scientific laws that describe the wacky behavior of photons, electrons and the other particles that make up the universe. It helps us understand the nature and behavior of matter and energy on the atomic and subatomic level [1].

Quantum mechanics include two independent formulations. The first formulation, called matrix mechanics, was developed by Heisenberg (1925) to describe atomic structure starting from the observed spectral lines. Inspired by Planck's quantization of waves and by Bohr's model of the hydrogen atom, Heisenberg founded his theory on the notion that the only allowed values of energy exchange between microphysical systems are those that are discrete: quanta. Expressing dynamical quantities such as energy, position, momentum and angular momentum in terms of matrices, he obtained an eigenvalue problem that describes the dynamics of microscopic systems; the diagonalization of the Hamiltonian matrix yields the energy spectrum and the state vectors of the system. Matrix mechanics was very successful in accounting for the discrete quanta of light emitted and absorbed by atoms.

The second formulation, called wave mechanics, was due to Schrödinger (1926); it is a generalization of the de Broglie postulate. This method, more intuitive than matrix mechanics, describes the dynamics of microscopic matter by means of a wave equation, called the Schrodinger equation; instead of the matrix eigenvalue problem of Heisenberg, Schrödinger obtained a differential equation. The solutions of this equation yield the energy spectrum and the wave function of the system under consideration. In 1927 Max Born proposed his probabilistic interpretation of wave mechanics: he took the square moduli of the wave functions that are solutions to the Schrodinger equation and he interpreted them as probability densities $[2,3]$.

These two ostensibly different formulations Schrödinger's wave formulation and Heisenberg's matrix approach were shown to be equivalent. Dirac then suggested a more general formulation of quantum mechanics which deals with abstract objects such as kets (state vectors), bras, and operators. The representation of Dirac's formalism in a continuous basis the position or momentum representations gives back Schrödinger's wave mechanics. As for Heisenberg's matrix formulation, it can be obtained by representing Dirac's formalism in a discrete basis. In this context, the approaches of Schrödinger and Heisenberg represent, respectively, the wave formulation and the matrix formulation of the general theory of quantum mechanics.

Combining special relativity with quantum mechanics, Dirac derived in 1928 an equation which describes the motion of electrons. This equation, known as Dirac's equation, predicted the existence of an antiparticle, the positron, which has similar properties, but opposite charge, with the electron; the positron was discovered in 1932, four years after its prediction by quantum mechanics.

In summary, quantum mechanics is the theory that describes the dynamics of matter at the microscopic scale. Fine! But is it that important to learn? This is no less than an otiose question, for quantum mechanics is the only valid framework for describing the microphysical world.

It is vital for understanding the physics of solids, lasers, semiconductor and superconductor devices, plasmas, etc. In short, quantum mechanics is the founding basis of all modern physics: solid state, molecular, atomic, nuclear, and particle physics, optics, thermodynamics, statistical mechanics, and so on. Not only that, it is also considered to be the foundation of chemistry and biology.

Despite the remarkable successes of quantum equations, but they suffer from noticeable set backs. For example, the quantum equation can not differentiate between the behavior of two particles subjected to the same potential, but one moves in free space and the other moves inside matter. This is in direct conflict whit experimental observations. Thus one needs new quantum equation that differentiates between 


\section{International Journal of Science and Research (IJSR) \\ ISSN (Online): 2319-7064}

Index Copernicus Value (2013): 6.14 | Impact Factor (2015): 6.391

the two situations. This is done in section(2). Section (3) is concerned with harmonic oscillator solution, sections (4) and (5) are the discussion and conclusion.

\section{Friction Effect on Momentum Term in Schrodinger Equation}

The ordinary quantum mechanical laws no terms feeling the effect of friction [4]. Recently some attempts made by M.Dirar and others[5,6] recognize the effect of friction on energy. This effect shows how energy and wave function decays with time due to friction effect.

When particle move in frictional medium, the frictional energy of it is given.

$$
E_{\mathrm{f}}=\left(\frac{\hbar}{\tau}\right) \mathrm{i}
$$

Where $\tau$ is the relaxation time.

To find the corresponding frictional momentum one can use special relativistic energy - momentum relation. According to this (SR) relation the relativistic frictional energy gives by

Thus using (1) and (2) yields:

$$
E_{\mathrm{f}}=C P_{\mathrm{f}}
$$

$$
P_{\mathrm{f}}=\frac{E_{\mathrm{f}}}{C}=\left(\frac{\hbar}{c \tau}\right) i
$$

Which is the momentum loss by friction .

For any system moving with velocity $\mathrm{v}$, the momentum is given by

$$
\mathrm{P}=\mathrm{mv}
$$

Thus the total momentum for frictional medium is given by

$$
\widetilde{P}=\mathrm{P}-\mathrm{P}_{\mathrm{f}}(5)
$$

For the situation in which there is both a kinetic energy and a potential present, the total energy of the system in Newtonian mechanics ( $S R$ for law speed) is given by

$\mathrm{E}=\frac{\left(\mathrm{P}-\mathrm{P}_{\mathrm{f}}\right)^{2}}{2 \mathrm{~m}}+\mathrm{V}$

Thus

$$
E=\frac{P^{2}}{2 m}-\frac{P_{f}}{m}+\frac{P_{f}^{2}}{2 m}+V
$$

Multiplying both sides of equation (6) by $\psi$, yields

$$
\mathrm{E} \psi=\frac{\mathrm{P}^{2}}{2 \mathrm{~m}} \psi-\frac{\mathrm{PP}_{\mathrm{f}}}{\mathrm{m}} \psi+\frac{\mathrm{P}_{\mathrm{f}}^{2}}{2 \mathrm{~m}} \psi+\mathrm{V} \psi
$$

Relation (7) can be used to find Schrodinger equation for particles moving in a resistive medium. This wave function for a free particle of momentum $\mathrm{P}$ and $\mathrm{E}$. This wave function is given by

$\psi=e^{\frac{i}{\hbar}(P x-E t)}$

$\frac{\partial \psi}{\partial x}=\frac{i P}{\hbar} e^{\frac{i}{\hbar}(P x-E t)}$

Thus

$\nabla \psi=\frac{\partial \psi}{\partial x}=\frac{i P}{\hbar} \psi$

$$
P \psi=\frac{\hbar}{i} \nabla \psi
$$

Differentiating again $\omega$. r.t $\mathrm{x}$ gives

$\nabla^{2} \psi=\frac{\partial^{2} \psi}{\partial x^{2}}=-\frac{P^{2}}{\hbar^{2}} e^{\frac{i}{\hbar}(P x-E t)}$

Hence

$\nabla^{2} \psi=\frac{\partial^{2} \psi}{\partial x^{2}}=-\frac{P^{2}}{\hbar^{2}} \psi$

$$
P^{2} \psi=-\hbar^{2} \nabla^{2} \psi
$$

The wave function can also be differentiated $\omega$. r.t $t$ to get

$$
\begin{aligned}
& \frac{\partial \psi}{\partial t}=-\frac{i}{\hbar} E e^{\frac{i}{\hbar}(P x-E t)} \\
& \frac{\partial \psi}{\partial t}=-\frac{i}{\hbar} E \psi \\
& E \psi=-\frac{\hbar}{i} \frac{\partial \psi}{\partial t}
\end{aligned}
$$

$$
E \psi=i \hbar \frac{\partial \psi}{\partial t}
$$

By Substituting equations (3),(8),(9) and (10) in equation

(7) the modified Schrodinger equation, yields

$$
i \hbar \frac{\partial \psi}{\partial t}=-\frac{\hbar^{2}}{2 m} \nabla^{2} \psi-\frac{\hbar^{2}}{\mathrm{mc} \tau} \nabla \psi-\frac{\hbar^{2}}{2 \mathrm{mc}^{2} \tau^{2}} \psi+\mathrm{V} \psi
$$

The coefficient of friction $\gamma$ is given by

$$
\gamma=\frac{\mathrm{m}}{\tau}
$$

Thus Schrodinger equation is given by

$$
i \hbar \frac{\partial \psi}{\partial t}=-\frac{\hbar^{2}}{2 m} \nabla^{2} \psi-\frac{\hbar^{2} \gamma}{\mathrm{m}^{2} \mathrm{c}} \nabla \psi-\frac{\hbar^{2} \gamma^{2}}{2 \mathrm{~m}^{3} \mathrm{c}^{2}} \psi+\mathrm{V} \psi
$$

In the absence of friction

$$
\gamma=0
$$

The equation (13) reduces to ordinary Schrodinger equation

$$
i \hbar \frac{\partial \psi}{\partial t}=-\frac{\hbar^{2}}{2 m} \nabla^{2} \psi+\mathrm{V} \psi
$$

The time independent Schrodinger equation (13) can be written in the form

$$
\mathrm{E} \psi=-\frac{\hbar^{2}}{2 m} \nabla^{2} \psi-\frac{\hbar^{2} \gamma}{\mathrm{m}^{2} \mathrm{c}} \nabla \psi-\frac{\hbar^{2} \gamma^{2}}{2 \mathrm{~m}^{3} \mathrm{c}^{2}} \psi+\mathrm{V} \psi
$$

\section{Harmonic Oscillator Solution}

Electrons moving in a circular orbit around the nucleus is a harmonic oscillator. This is since for such motion $\mathrm{F}=\mathrm{ma}=-\mathrm{m} \omega^{2} \mathrm{r}$

$$
\mathrm{V}=-\int \mathrm{Fds}=\mathrm{m} \omega^{2} \int \mathrm{r}(2 \pi \mathrm{rd} \theta)=\mathrm{m} \omega^{2}(2 \pi)^{2} \mathrm{r}^{2} \sim \mathrm{Kr} \mathrm{r}^{2}
$$

To find solution for harmonic oscillator, consider the wave function is given by

$$
\psi=e^{i k x} u(x)
$$

Which can be differentiated $\omega$. .r.t to $\mathrm{x}$ to get

$\nabla \psi=i k e^{i k x} u+e^{i k x} \nabla u$

$$
\nabla^{2} \psi=-k^{2} e^{i k x} u+2 i k e^{i k x} \nabla u+e^{i k x} \nabla^{2} u
$$

substituting equations (18) and (19) in equation (16) yields:

$$
\begin{aligned}
\frac{\hbar^{2} k^{2}}{2 m} u-\frac{i k \hbar^{2}}{m} \nabla u & -\frac{\hbar^{2}}{2 m} \nabla^{2} u-\frac{i k \hbar^{2}}{m c \tau} u-\frac{\hbar^{2}}{m c \tau} \nabla u \\
& -\frac{\hbar^{2}}{2 m C^{2} \tau^{2}} \mathrm{u}+\mathrm{Vu} \\
= & \mathrm{Eu}
\end{aligned}
$$

For

$$
\frac{i k \hbar^{2}}{m}=-\frac{\hbar^{2}}{m c \tau}
$$

The wave number is given by

$$
k=\frac{i}{c \tau}
$$

Substituting equations ( 21$)$ and (22) in equation ( 20$)$ ,yields :

$$
\begin{aligned}
-\frac{\hbar^{2}}{2 m} \nabla^{2} u+(- & \left.\frac{i k \hbar^{2}}{m}+\frac{i k \hbar^{2}}{m}\right) \nabla u \\
& +\left(\frac{\hbar^{2} k^{2}}{2 m}-\frac{\hbar^{2} k^{2}}{m}+\frac{\hbar^{2} k^{2}}{2 m}\right) u+\mathrm{Vu}=\mathrm{Eu}
\end{aligned}
$$

Thus

$$
-\frac{\hbar^{2}}{2 m} \nabla^{2} u+\mathrm{Vu}=\mathrm{Eu}
$$

Comparing this expression it is clear that this is typical to that of the ordinary harmonic oscillator. For Harmonic

$$
\mathrm{V}=\frac{1}{2} k x^{2}
$$




\section{International Journal of Science and Research (IJSR) \\ ISSN (Online): 2319-7064}

Index Copernicus Value (2013): 6.14 | Impact Factor (2015): 6.391

$$
\mathrm{E}=\left(n+\frac{1}{2}\right) \hbar \omega
$$

Which is the ordinary energy for oscillator in the absence of friction. However the wave function in equation(18) for $\mathrm{k}$ given by equation (22) takes the form:

$$
\psi=e^{-\frac{x}{c \tau}} u(x)
$$

which represents a decaying wave function this means that friction decreases the number of particles.

Consider now the solution

$$
\begin{aligned}
& u=C_{0} e^{\alpha x^{2}} \\
& \begin{array}{l}
\nabla u=2 \alpha x C_{0} e^{\alpha x^{2}}=2 \alpha x u \\
\nabla^{2} u=\left(2 \alpha+4 \alpha^{2} x^{2}\right) u
\end{array}
\end{aligned}
$$

Substituting equations ( 24),(25) and (26) in equation (23) ,yields :

$$
\begin{aligned}
-\frac{\hbar^{2}}{2 m}(2 \alpha+ & \left.4 \alpha^{2} x^{2}\right) u+\frac{1}{2} k x^{2} u=\left(n+\frac{1}{2}\right) \hbar \omega u \\
- & \frac{\hbar^{2}}{2 m}\left(2 \alpha+4 \alpha^{2} x^{2}\right)+\frac{1}{2} k x^{2}=\left(n+\frac{1}{2}\right) \hbar \omega
\end{aligned}
$$

Comparing the free terms and coefficients of $x^{2}$ on both sides yields:

$$
\begin{array}{ll}
-\frac{2 \hbar^{2} \alpha^{2}}{m}+\frac{1}{2} k=0 & \\
\alpha^{2}=\frac{m}{4 \hbar^{2}} k & \alpha=\frac{\sqrt{m k}}{2 \hbar}
\end{array}
$$

Thus

$$
\begin{aligned}
& -\frac{\hbar^{2}}{m} \alpha=\left(n+\frac{1}{2}\right) \hbar \omega \\
& \alpha=-\frac{m}{\hbar}\left(n+\frac{1}{2}\right) \omega
\end{aligned}
$$

Thus from equation (29) and (30) :

$$
\begin{aligned}
& \frac{\sqrt{m k}}{2 \hbar}=-\frac{m}{\hbar}\left(n+\frac{1}{2}\right) \omega \\
& k=2 m\left(n+\frac{1}{2}\right)^{2} \omega^{2}
\end{aligned}
$$

$$
m=\frac{k}{2\left(n+\frac{1}{2}\right)^{2} \omega^{2}}
$$

This means that The mass is quantized

\section{Discussion}

The difference between matter and free space manifests itself through frictional term in the expression of momentum as shown by equation (3). Two terms recognize friction, according to equation(11). This equation reduces to ordinary Schrodinger equation in the absence of friction(see equation(15)).

Since string theory treats particles as harmonic oscillators it is thus quite obvious to try harmonic solutions. Suggesting solution equation (18) for the spatially oscillating system, the wave number is imaginary according to equation(22). This leads to spatially decaying wave function (26). This wave function (26). This wave function can describe inelastic scattering press in which the number of particles in a beam is reduced, where

$$
\mathrm{n} \sim|\psi|^{2}=e^{-\frac{2 x}{c \tau}}
$$

It is very interesting to note that the energy $\mathrm{E}$ equation(25) shows no change of energy per particle. This means that energy loss due to friction changes the number of particles and does not change the energy of a single particle. This resembles inelastic scattering, which leads to atomic excitation that changes their energy by an amount $(\hbar \omega)$. Here the increase or decrease due to friction and collision is caused by the change of the number of excited atoms.

\section{Conclusion}

The effect of friction on Schrodinger equation leads to describing the inelastic scattering process. It shows that in the elastic scattering number of particles is not conserved references

\section{Acknowledgments}

We would like to thank all those who encouraged or assisted them in this work.

\section{References}

[1] David Morin, Introduction to quantum Mechanics, Cambridge University Press, uk,2008.

[2] Nouredine Zettili, Quantum Mechanics Concepts and Applications, Jacksonville State University, Jacksonville, USA, 2009.

[3] G. Aruldhas, Quantum Mechanics, PHI leaming private limited. New Delhi, 2009.

[4] L.I. Schiff, Quantum Mechanics ,Mc Graw Hill, Tokyo, 2015

[5] Mobarak Ibrahim etal, IJESRT, V.4, ISSN: 2277-9655, 2015

[6] Lutfi etal , IJESRT,V.5, ISSN: 2277-9655, 2016. 